\title{
Important Docking study of Structure of Plasmodium falciparum thioredoxin reductase-thioredoxin complex: the case of potent drug Telatinib, a small molecule angiogenesis inhibitor
}

\author{
Ivan Vito Ferrari 1* \\ 1. University of Rome Tor Vergata, Department of Industrial Engineering, Via del Politecnico 1, 00133 \\ Rome, Italy; Ivan.Vito.Ferrari@uniroma2.it
}

Corresponding Author: Ivan.Vito.Ferrari@uniroma2.it

Keywords: Plasmodium falciparum, Autodock 4, Autodock Vina

\begin{abstract}
Background: Over the last decades, malaria parasites have been rapidly developing resistance against antimalarial drugs, which underlines the need for novel drug targets. Thioredoxin reductase (TrxR) is crucially involved in redox homeostasis and essential for Plasmodium falciparum. In this communication, we report first time important Docking study by in Silico approach, using AutoDock Vina. After a selective analysis of over 300 drugs, processed with Pyrx (a Virtual Screening software into the active site of protein (ID PDB 4J56 Thioredoxin reductase 2 Chain A), we noticed excellent value of Binding Energy of Telatinib estimated by Pyrx software. These results are comparable to the crystallized ligand FAD ( FLAVIN-ADENINE DINUCLEOTIDE) completed in the above-mentioned protein. Indeed, from the results of Autodock Vina, Telatinib an inhibitor of tyrosine kinases, has excellent a Binding affinity value, ca. $-12 \mathrm{kcal} / \mathrm{mol}$.

\section{Introduction}

Over the last decades, malaria parasites have been rapidly developing resistance against antimalarial drugs, which underlines the need for novel drug targets. Thioredoxin reductase (TrxR) is crucially involved in redox homeostasis and essential for Plasmodium falciparum[1] .Plasmodium falciparum is a unicellular protozoan parasite of humans, and the deadliest species of Plasmodium that causes malaria in humans. The parasite is transmitted through the bite of a female Anopheles mosquito and causes the disease's most dangerous form, falciparum malaria [2] . It is responsible for around $50 \%$ of all malaria cases. Plasmodium falciparum is the Plasmodium species responsible for $85 \%$ of the malaria cases. Malaria infects over 200 million people annually, mostly in poor tropical and subtropical countries of Africa [2-3] . In this short communication, we investigated about 300 drugs, through In Silico Docking approach, downloaded from PubChem Database ( https://pubchem.ncbi.nlm.nih.gov/). We have focused on Autodock Vina, estimated with Pyrx software, a simple Virtual Screening library software for Computational Drug Discovery ( https://pyrx.sourceforge.io/), based on prediction the binding orientation and affinity of a ligand [4].
\end{abstract}


bioRxiv preprint doi: https://doi org/10.1101/2021.07 24 453649. this version posted July 26, 2021. The copyriaht holder for this preprin (which was not certified by peer review) is the author/funder, who has granted bioRxiv a license to display the preprint in perpetuity. It is made available under aCC-BY-NC 4.0 International license.

\section{Materials and methods}

\subsection{Protein and Ligand Preparation before docking}

\section{Protein Preparation:}

4J56 [Structure of Plasmodium falciparum thioredoxin reductase-thioredoxin complex: Thioredoxin reductase 2] was prepared manually using several software, before molecular docking analysis. The first step ,was downloaded from Protein Data Bank, https://www.rcsb.org/structure/4j56and save in pdb format. The second step were removed all unnecessary docking chains. In fact, in this case we are only Chain A ( https://www.rcsb.org/structure/6LD3) has been maintained and re-saved in pdb format. ( See below figure 1) Next, were the removal of ligands and water molecules crystallized using Chimera software [ 6]. Later, polar hydrogens and Kollmann charges were added with Mgl-Tool, ( or called AutoDockTools, a software developed at the Molecular Graphics Laboratory (MGL) [7] As a last step they were added to the protein, any missing amino acids and the whole protein was minimized with the Swiss PDB Viewer Software [8].

Ligand Preparation: the first step, was to separate the crystallized ligand G80 ( 2,4-dimethoxy-5-thiophen-2-ylbenzoic acid) from its protein, manually add all the hydrogens and their Gasteiger charges (with the MGL Tool software) and minimize it with MMFF94 force field [9], and opened by Pyrx software ( https://pyrx.sourceforge.io/) .

Parameters Grid Box for Docking in Ligand Binding Site Pocket for Repeatability Binding Affinity by AMDock Software calculated with Autodock Vina and Autodock 4:

- ID PDB 4J56: Structure of Plasmodium falciparum thioredoxin reductase-thioredoxin complex Chain A: Center X (= -32.19); Centre Y (=-109.21); Centre Z (=196.34); Dimensions (Angstrom) ( $\AA$ ) X, Y, Z [= $26.70=, 25.00,=20.53]$; exhaustiveness $=8$.

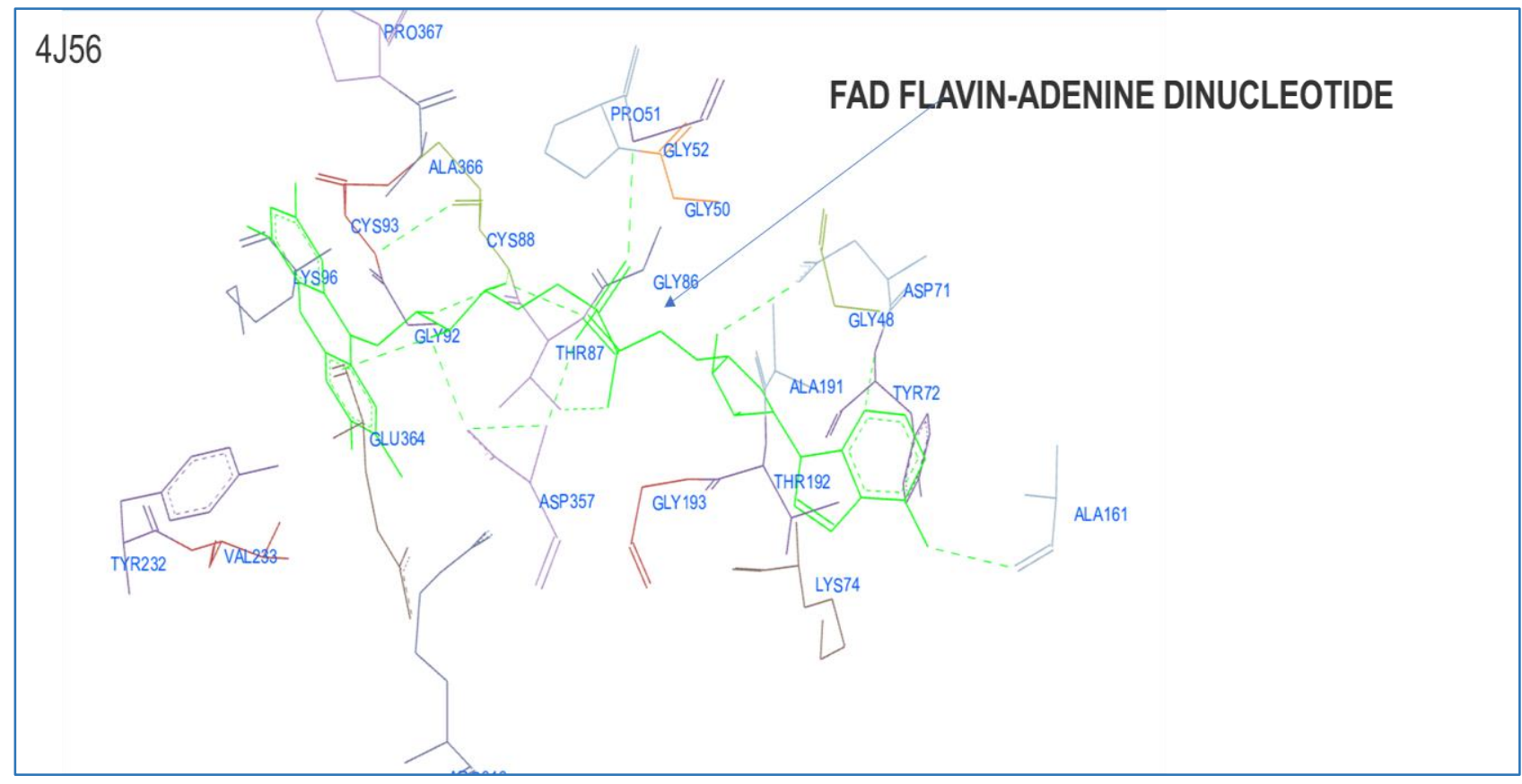

Fig 13D Structure of ID PDB 4J56 Chain A (Structure of Plasmodium falciparum thioredoxin reductase-thioredoxin complex by Discovery Studio Biovia Software [10] 


\section{Discussion and Results}

In this communication, we investigated about 300 drugs, through In Silico Docking approach, downloaded from PubChem Database ( https://pubchem.ncbi.nlm.nih.gov/). From our results of Autodock Vina, estimated with Pyrx software, a simple Virtual Screening library software for Computational Drug Discovery ( https://pyrx.sourceforge.io/), based on prediction the binding orientation and affinity of a ligand [ 4] ( See below table 1) we have selected only best Binding Energy of drugs ( $\mathrm{kcal} / \mathrm{mol}$ ) ( See below table 2). After intensive docking investigation by Autodock Vina, we propose a potential candidate drug against Malaria, that is Telatinib , $\mathrm{C}_{20} \mathrm{H}_{16} \mathrm{CIN}_{5} \mathrm{O}_{3}$. ( See below figure 2) It is an orally administered drug that inhibits VEGFR-2, VEGFR3 PDGFR- $\beta$ and c-Kit (Eskens et al., 2009) and this drug is an orally available, potent multitargeted VEGFR-2, VEGFR-3, PDGFR- $\beta$ and c-Kit tyrosine kinases inhibitor with an IC50 of $19 \mathrm{nM}$ for the inhibition of VEGFR-2 autophosphorylation and it is under investigation in clinical trial NCT03817411 (Telatinib in Combination With Capecitabine/ Oxaliplatin in 1st Line Gastric or GEJ Cancer [11-12]. We noticed excellent value of Binding Energy $(\mathrm{kcal} / \mathrm{mol})$ of Telatinib by docking analysis. These results are are comparable to the crystallized ligand FAD ( FLAVIN-ADENINE DINUCLEOTIDE) completed in the above-mentioned protein. Indeed, from the results of Autodock Vina, estimated by Pyrx, Telatinib has excellent Binding affinity value, ca. $-12 \mathrm{kcal} / \mathrm{mol}$. ( See below Table 2).

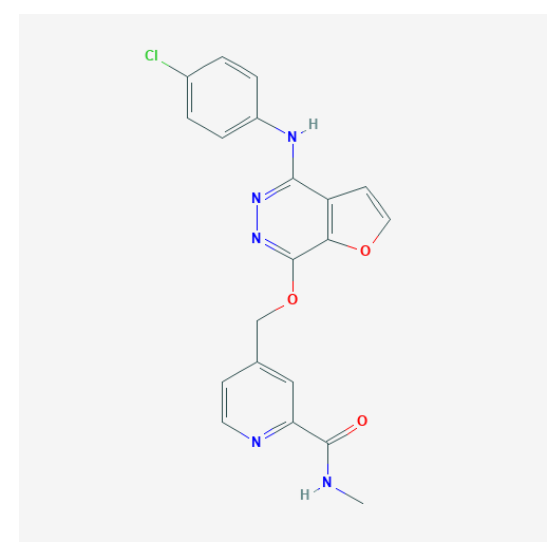

Fig.2 Structure of Telatinib 
bioRxiv preprint doi: https://doi.org/10.1101/2021.07.24.453649; this version posted July 26, 2021. The copyright holder for this preprint (which was not certified by peer review) is the author/funder, who has granted bioRxiv a license to display the preprint in perpetuity. It is made available under aCC-BY-NC 4.0 International license.

\begin{tabular}{|c|c|c|c|}
\hline Ligand & Binding Energy (kcal/mol) & Ligand & Binding Energy (kcal/ $/ \mathrm{mol})$ \\
\hline Abacavir_mmff94_E=-36.60 & -8.9 & Relacatib_mmff94_E=97.80 & -10.1 \\
\hline Abemaciclib_mmff94_E=60.13 & -9.3 & Remdesivir_mmff94_E=-55.49 & -8.8 \\
\hline Abiraterone_mmff94_E=109.51 & -8.5 & Repaglinide_mmff94_E $=55.46$ & -8.2 \\
\hline Abivertinib_mmff94_E=73.31 & -9.1 & Ribavirin_mmff94_E=141.90 & -8.1 \\
\hline Acalabrutinib_mmff94_E=-56.44 & -9.0 & Ritonavir_mmff94_E=-31.38 & -8.6 \\
\hline Acamprosate_mmff94_E=-79.96 & -5.8 & Roflumilast_mmff94_E=119.06 & -8.5 \\
\hline Acarbose_mmff94_E=363.05 & -7.5 & Rucaparib_mmff94_E=28.81 & -8.2 \\
\hline Aceclofenac_mmff94_E=67.36 & -6.6 & Ruxolitinib_mmff94_E=14.83 & -9.8 \\
\hline Acenocoumarol_mmff94_E=86.55 & -7.8 & Selinexor_mmff94_E=150.82 & -11.3 \\
\hline Acetaminophen_mmff94_E $=-12.21$ & -6.3 & Sepetaprost_mmff94_E=92.24 & -10.1 \\
\hline Acetazolamide_mmff94_E=-59.56 & -7.0 & Simvastatin_mmff94_E=33.89 & -7.9 \\
\hline Acyclovir_mmff94_E=-35.68 & -7.2 & Sirolimus_mmff94_E=287.84 & 1.1 \\
\hline Alfacalcidol_mmff94_E= $=82.13$ & -7.8 & Sitagliptin_mmff94_E=41.81 & -10.0 \\
\hline Alfatradiol_mmff94_E=59.38 & -7.5 & Sonidegib_mmff94_E=111.94 & -10.3 \\
\hline Ambroxol_mmff94_E=27.84 & -7.1 & Sontoquine_mmff94_E=45.27 & -7.4 \\
\hline Amcinonide_mmff94_E= 159.87 & -8.6 & Stavudine_mmff94_E=-71.37 & -6.9 \\
\hline Amikacin_mmff94_E=308.55 & -6.0 & Sufentanil_mmff94_E=70.52 & -6.8 \\
\hline Aminopterin_mmff94_E=-30.98 & -10.1 & Sunitinib_mmff94_E=38.30 & -8.3 \\
\hline Aminoquinol_mmff94_E=60.49 & -8.5 & Tacalcitol_mmff94_E=88.34 & -6.5 \\
\hline Amoxicillin_mmff94_E=78.37 & -7.7 & Tadalafil_mmff94_E=94.12 & -7.7 \\
\hline Ampicillin_mmff94_E=90.57 & -8.0 & Tafetinib_mmff94_E=43.22 & -6.0 \\
\hline Amprenavir_mmff94_E=-9.35 & -8.9 & Tafluprost_mmff94_E=92.17 & -8.6 \\
\hline Anagrelide_mmff94_E=-73.24 & -7.9 & Tamoxifen_mmff94_E=106.43 & -5.9 \\
\hline Anakinra_mmff94_E=69.33 & -8.7 & Tarenflurbil_mmff94_E=47.36 & -8.2 \\
\hline Apremilast_mmff94_E=-1.94 & -8.2 & Taribavirin_mmff94_E=123.38 & -7.7 \\
\hline Aprepitant_mmff94_E=177.68 & -7.2 & Telaprevir_mmff94_E=210.06 & -6.0 \\
\hline Apricitabine_mmff94_E=-36.38 & -6.6 & Telatinib_mmff94_E=73.64 & -12.1 \\
\hline Aranidipine_mmff94_E=58.77 & -6.1 & Teniposide_mmff94_E=141.52 & -6.3 \\
\hline Arbidol_mmff94_E=61.66 & -5.7 & Tetrophan_mmff94_E=42.44 & -8.2 \\
\hline Aspirin_mmff94_E=20.42 & -6.7 & Thalidomide_mmff94_E=-42.10 & -8.4 \\
\hline Aspoxicillin_mmff94_E=53.65 & -7.8 & Thiamiprine_mmff94_E=-49.81 & -7.1 \\
\hline Atazanavir_mmff94_E=106.90 & -7.7 & Thymoquinone_mmff94_E=4.81 & -6.5 \\
\hline Atenolol_mmff94_E=58.74 & -7.9 & Ticlopidine_mmff94_E=46.13 & -7.4 \\
\hline Atomoxetine_mmff94_E=53.08 & -7.3 & Timolol_mmff94_E=108.32 & -7.5 \\
\hline Azithromycin_mmff94_E=305.98 & -6.1 & Tobramycin_mmff94_E=281.53 & -5.0 \\
\hline Baclofen_mmff94_E=4.56 & -6.8 & Toceranib_mmff94_E=38.51 & -10.5 \\
\hline Baricitinib_mmff94_E=-45.99 & -9.7 & Tolvaptan_mmff94_E=90.96 & -6.3 \\
\hline Bemcentinib_mmff94_E=85.13 & -11.4 & Torsemide_mmff94_E=-159.76 & -8.3 \\
\hline Bendazac_mmff94_E=54.84 & -8.9 & Trandolapril_mmff94_E=116.91 & -8.2 \\
\hline Betamethasone_mmff94_E $=187.30$ & -8.1 & Travoprost_mmff94_E=95.75 & -9.7 \\
\hline Bicalutamide_mmff94_E=64.96 & -10.5 & Traxoprodil_mmff94_E=73.05 & -8.5 \\
\hline Bictegravir_mmff94_E=34.41 & -9.6 & Treprostinil_mmff94_E=59.45 & -7.1 \\
\hline Bilirubin_mmff94_E=-40.94 & -9.3 & Triamcinolone_mmff94_E=190.64 & -6.9 \\
\hline Biliverdin_mmff94_E=-36.94 & -8.0 & Triazavirin_mmff94_E=98.77 & -7.8 \\
\hline Boceprevir_mmff94_E $=55.00$ & -6.5 & Triflusal_mmff94_E=43.56 & -7.6 \\
\hline Brensocatib_mmff94_E=106.46 & -11.3 & Ubrogepant_mmff94_E=95.91 & -9.1 \\
\hline Brequinar_mmff94_E=63.14 & -10.6 & Urapidil_mmff94_E=15.18 & -9.3 \\
\hline Brigatinib_mmff94_E=78.14 & -7.3 & Ursodeoxycholic_acid_mmff94_E=68.15 & -6.2 \\
\hline Buciclovir_mmff94_E=-47.53 & -7.5 & Valacyclovir_mmff94_E=1.25 & -8.3 \\
\hline Budesonide_mmff94_E=134.30 & -7.1 & Valsartan_mmff94_E=69.88 & -7.6 \\
\hline Buprenorphine_mmff94_E=180.32 & -6.8 & Vamorolone_mmff94_E=132.02 & -7.2 \\
\hline Cabotegravir_mmff94_E=73.64 & -9.8 & Verapamil_mmff94_E=141.23 & -8.6 \\
\hline Cabozantinib_mmff94_E=63.05 & -10.9 & Verdinexor_mmff94_E=94.79 & -11.4 \\
\hline Calcifediol_mmff94_E=90.84 & -7.2 & Vidofludimus_mmff94_E=-5.45 & -8.8 \\
\hline Calcipotriol_mmff94_E=90.21 & -8.1 & Vildagliptin_mmff94_E=76.54 & -9.5 \\
\hline Calcitriol_mmff94_E=89.98 & -7.8 & Vorapaxar_mmff94_E=-2.25 & -9.7 \\
\hline Camostat_mmff94_E=-44.38 & -9.7 & Vortioxetine_mmff94_E=136.53 & -6.2 \\
\hline Cangrelor_mmff94_E=-150.61 & -8.4 & Zalcitabine_mmff94_E=-63.62 & -6.5 \\
\hline Capecitabine_mmff94_E=-33.67 & -9.1 & Zanamivir_mmff94_E=-66.63 & -7.2 \\
\hline Carboxymefloquine_mmff94_E=73.73 & -7.5 & Zidovudine_mmff94_E=-71.00 & -7.9 \\
\hline Cefadroxil_mmff94_E=63.22 & -7.9 & Zuclopenthixol_mmff94_E=161.36 & -8.0 \\
\hline Cefamandole_mmff94_E=57.60 & -8.7 & lig_mmff94_E=-123.75 & -12.3 \\
\hline Cefdinir_mmff94_E=74.50 & -8.3 & Cinchophen_mmff94_E=36.78 & -9.3 \\
\hline Cefixime_mmff94_E=73.93 & -7.8 & Clobazam_mmff94_E=59.53 & -7.9 \\
\hline Cefonicid_mmff94_E=-9.97 & -9.1 & Clobetasol_mmff94_E=174.79 & -7.2 \\
\hline Cefotaxime_mmff94_E=20.15 & -6.7 & Clopenthixol_mmff94_E=161.27 & -8.3 \\
\hline Cefpodoxime_mmff94_E=37.12 & -6.8 & Cloperidone_mmff94_E=34.14 & -9.9 \\
\hline Cefprozil_mmff94_E=81.97 & -8.2 & Cobicistat_mmff94_E=5.31 & -8.4 \\
\hline Ceftizoxime_mmff94_E=42.12 & -7.0 & Cobimetinib_mmff94_E=116.08 & -9.1 \\
\hline Cenicriviroc_mmff94_E=126.10 & -6.1 & Codeine_mmff94_E=116.60 & -7.6 \\
\hline Censavudine_mmff94_E=-60.94 & -7.7 & Colchicine_mmff94_E=108.54 & -6.3 \\
\hline Ceritinib_mmff94_E=30.54 & -7.7 & Colchine_mmff94_E=109.57 & -6.9 \\
\hline Chloroquine_mmff94_E=35.47 & -6.9 & Cortisone_mmff94_E=119.79 & -7.5 \\
\hline Ciclesonide_mmff94_E=118.35 & -7.6 & Cortodoxone_mmff94_E=124.34 & -7.1 \\
\hline Dehydrocorticosterone_mmff94_E=94.: & -7.5 & Crizotinib_mmff94_E=25.76 & -9.2 \\
\hline Delorazepam_mmff94_E=102.75 & -7.6 & Cytarabine_mmff94_E $=0.10$ & -7.0 \\
\hline Desethylchloroquine_mmff94_E=26.57 & -7.1 & Cytidine_mmff94_E=0.70 & -7.0 \\
\hline Desogestrel_mmff94_E=80.85 & -7.2 & D-Urobilinogen_mmff94_E $=-61.78$ & -8.7 \\
\hline Desonide_mmff94_E=136.79 & -7.5 & Dabigatran_mmff94_E=-17.44 & -9.8 \\
\hline Desoximetasone_mmff94_E=151.31 & -7.5 & Darunavir_mmff94_E=-16.81 & -8.4 \\
\hline Dexamethasone_mmff94_E=185.43 & -7.7 & Dasatinib_mmff94_E=39.32 & -9.6 \\
\hline Dexketoprofen_mmff94_E=73.36 & -8.3 & Dihydromorphine_mmff94_E=95.03 & -7.2 \\
\hline Dexlansoprazole_mmff94_E $=77.20$ & -9.1 & Diltiazem_mmff94_E=106.16 & -6.9 \\
\hline Diclofenac_mmff94_E=57.54 & -6.5 & Docetaxel_mmff94_E=212.08 & -6.5 \\
\hline Dihydroergotamine_mmff94_E=86.25 & -8.3 & Dolutegravir_mmff94_E=42.86 & -9.8 \\
\hline Duraspiron_mmff94_E=79.94 & -7.4 & Doxercalciferol_mmff94_E=89.95 & -7.1 \\
\hline Dutasteride_mmff94_E=101.30 & -7.8 & Doxorubicin_mmff94_E=181.35 & -5.8 \\
\hline Econazole_mmff94_E=49.35 & -7.7 & Ebastine_mmff94_E=113.72 & -9.5 \\
\hline Efavirenz_mmff94_E=15.90 & -6.6 & & \\
\hline
\end{tabular}

Table 1 Binding energies ( Kcal/mol) of Drugs into the active site of ID PDB $4 J 56$ protein estimated by Autodock Vina with Pyrx 
bioRxiv preprint doi: https://doi.org/10.1101/2021.07.24 453649. this version posted July 26, 2021. The copyright holder for this preprin (which was not certified by peer review) is the author/funder, who has granted bioRxiv a license to display the preprint in perpetuity. It is made available under aCC-BY-NC 4.0 International license.

\begin{tabular}{|l|c|}
\hline \multicolumn{1}{|c|}{ Ligand } & Binding Energy $(\mathrm{kcal} / \mathrm{mol})$ \\
\hline Bemcentinib_mmff94_E=85.13 & $\mathbf{- 1 1 . 4}$ \\
\hline Brequinar_mmff94_E=63.14 & $\mathbf{- 1 0 . 6}$ \\
\hline Cabozantinib_mmff94_E=63.05 & $\mathbf{- 1 0 . 9}$ \\
\hline lig_mmff94_E=-123.75 & -12.3 \\
\hline Selinexor_mmff94_E=150.82 & $\mathbf{- 1 1 . 3}$ \\
\hline Telatinib_mmff94_E=73.64 & -12.1 \\
\hline Verdinexor_mmff94_E=94.79 & $\mathbf{- 1 1 . 4}$ \\
\hline
\end{tabular}

Table 2 Best Binding energies ( Kcal/mol) of Drugs into the active site of ID PDB 4J56 protein estimated by Autodock Vina with Pyrx software

\section{ID PDB 4J56 : Structure of Plasmodium falciparum thioredoxin reductase-thioredoxin complex}

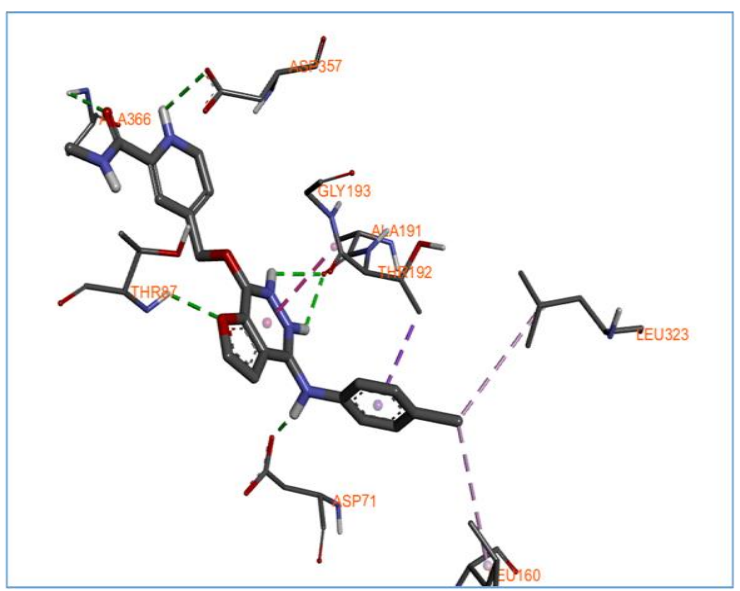

$-12.01 \mathrm{kcal} / \mathrm{mol}$ Telatinib

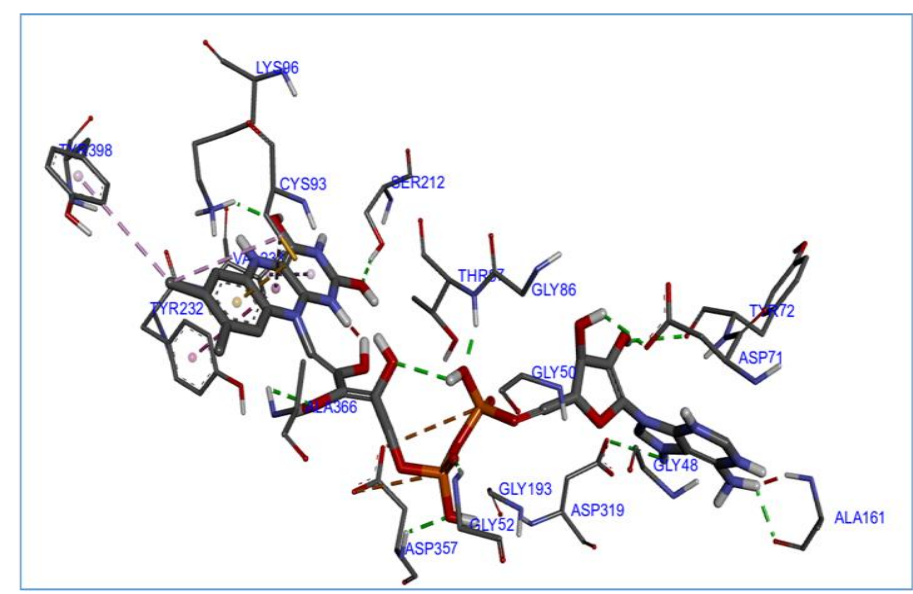

-12.03 kcal/mol crystallized FAD (FLAVINADENINE DINUCLEOTIDE)

Fig. 3 Comparison Binding Energy kcal/mol of Crystalized FAD - 12,0 kcal/mol with proposed drug Telatinib -12,01 kcal/ml into the active site of protein ID PDB: "Structure of Plasmodium falciparum thioredoxin reductase-thioredoxin complex", calculated by Autodock Vina with Pyrx software. This figure was produced by Discovery Studio Biovia Software [10]

\section{Conclusion}

In this communication, we report first time important Docking study by in Silico approach, using AutoDock Vina. After a selective analysis of over 300 drugs, processed with Pyrx (a Virtual Screening software into the active site of protein ( ID PDB 4J56 Thioredoxin reductase 2 Chain A), we noticed excellent value of Binding Energy of Telatinib estimated by Pyrx software. These results are comparable to the crystallized ligand FAD ( FLAVIN-ADENINE DINUCLEOTIDE) completed in the above-mentioned protein. Indeed, from the results of Autodock Vina, Telatinib an inhibitor of tyrosine kinases, has excellent a Binding affinity value, ca. -12.01 $\mathrm{kcal} / \mathrm{mol}$ versus $-12.03 \mathrm{kcal} / \mathrm{mol}$ of crystallized ligand FAD.

\section{Author contributions}

I.V.F. conceived, designed and wrote the paper, performed the calculations and analysed the data.

\section{Declaration of Competing Interest}

The authors declare they have no potential conflicts of interest to disclose. 


\section{$\underline{\text { REFERENCES }}$}

1. Fritz-Wolf, K., Jortzik, E., Stumpf, M., Preuss, J., lozef, R., Rahlfs, S., \& Becker, K. (2013). Crystal structure of the Plasmodium falciparum Thioredoxin reductase-Thioredoxin complex. Journal of molecular biology, 425(18), 3446-3460.

2. Antinori, S., Galimberti, L., Milazzo, L., \& Corbellino, M. (2012). Biology of human malaria plasmodia including Plasmodium knowlesi. Mediterranean journal of hematology and infectious diseases, 4(1).

3. Jortzik E, Becker K. Thioredoxin and glutathione systems in Plasmodium falciparum. Int J Med Microbiol. 2012 Oct;302(4-5):187-94. doi: 10.1016/j.ijmm.2012.07.007. Epub 2012 Aug 29. PMID: 22939033

4. Trott, Oleg, and Arthur J. Olson. "AutoDock Vina: improving the speed and accuracy of docking with a new scoring function, efficient optimization, and multithreading." Journal of computational chemistry 31.2 (2010): 455-461.

5. Morris, G. M., Huey, R., Lindstrom, W., Sanner, M. F., Belew, R. K., Goodsell, D. S. and Olson, A. J. (2009) Autodock4 and AutoDockTools4: automated docking with selective receptor flexiblity. J. Computational Chemistry 2009, 16: 2785-91.

6. Pettersen, Eric F., et al. "UCSF Chimera-a visualization system for exploratory research and analysis." Journal of computational chemistry 25.13 (2004): 1605-1612.

7. http://mgltools.scripps.edu/

8. Guex, Nicolas, and Manuel C. Peitsch. "SWISS-MODEL and the Swiss-Pdb Viewer: an environment for comparative protein modeling." electrophoresis 18.15 (1997): 2714-2723.

9. Thomas A. Halgren, J. Comput. Chem., 17, 490-519 (1996).

10. Biovia, Dassault Systèmes. "BIOVIA workbook, release 2017; BIOVIA pipeline pilot, release 2017." San Diego: Dassault Systèmes (2020).

11. Steeghs, N., Gelderblom, H., Op't Roodt, J., Christensen, O., Rajagopalan, P., Hovens, M., ... \& de Koning, E. (2008). Hypertension and rarefaction during treatment with telatinib, a small molecule angiogenesis inhibitor. Clinical Cancer Research, 14(11), 3470-3476.

12. Bronte, E., Galvano, A., Novo, G., \& Russo, A. (2016). Cardiotoxic effects of anti-VEGFR tyrosine kinase inhibitors. 